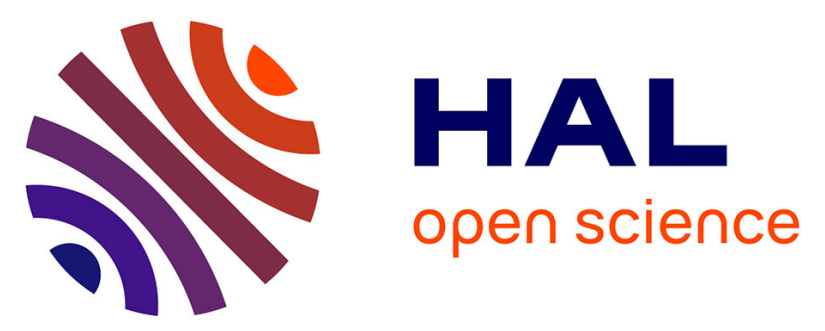

\title{
Repeatability and Reproducibility Analysis of a Multistable Module Devoted to Digital Microrobotics
}

Ismail Bouhadda, Abdenbi Mohand-Ousaid, Gilles Bourbon, Patrice Le Moal, Philippe Lutz, Hussein Hussein, Yassine Haddab

\section{- To cite this version:}

Ismail Bouhadda, Abdenbi Mohand-Ousaid, Gilles Bourbon, Patrice Le Moal, Philippe Lutz, et al.. Repeatability and Reproducibility Analysis of a Multistable Module Devoted to Digital Microrobotics. IROS: Intelligent Robots and Systems, Oct 2018, Madrid, Spain. pp.4889-4894, 10.1109/IROS.2018.8594259 . lirmm-02006666

\section{HAL Id: lirmm-02006666 \\ https://hal-lirmm.ccsd.cnrs.fr/lirmm-02006666}

Submitted on 4 Feb 2019

HAL is a multi-disciplinary open access archive for the deposit and dissemination of scientific research documents, whether they are published or not. The documents may come from teaching and research institutions in France or abroad, or from public or private research centers.
L'archive ouverte pluridisciplinaire HAL, est destinée au dépôt et à la diffusion de documents scientifiques de niveau recherche, publiés ou non, émanant des établissements d'enseignement et de recherche français ou étrangers, des laboratoires publics ou privés. 


\title{
Repeatability and reproducibility analysis of a multistable module devoted to digital microrobotics
}

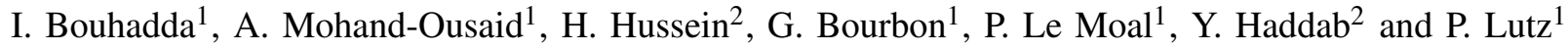

\begin{abstract}
The digital microrobot, called DiMiBot, opened a new paradigm in the design of microrobots by using mechanical stability instead of complex control strategies. Current DiMiBot robots are based on the use of bistable modules to reach discrete stable positions. However, the number of stable positions depends on the number of bistable modules. As a consequence, the mechanism size increases rapidly and its miniaturization becomes complex and non-intuitive. To tackle this issue, a new multistable module has been developed to reach several stable positions within a miniaturized structure. In this paper, we focus on the reapitability and the reproducibility analysis of the developed multistable module in terms of displacement. This study is mandatory to demonstrate the effectiveness of the module as it is expected to be an elementary component of the next generation of DiMiBot. To this end, a series of experimental measurements are conducted on individual and multiple modules. The results analysis show a good agreement between the theoretical and the experimental displacements. In other words, the multistable prototype is able to reach 13 stable positions linearly in one dimensional direction with a step of about $10 \mu \mathrm{m}$. These capabilities open a promising perspectives and applications of this module to achieve microrobotics tasks. For example, it can be integrated in complex systems devoted to advanced tasks or accurate positioning in MEMS devices.
\end{abstract}

\section{INTRODUCTION}

To perform tasks in the Microworld, i.e. the world of submillimetric objects, in particular micromanipulation, characterization or micro-assembly of objects, specific robotic systems are required. Thanks to the progress in the field of micro/nanotechnologies and smart materials, several types of microrobots were proposed. Most of them employ an actuation mode to provide a precise positioning which is essential to achieve robotic tasks. Generally, two of those modes are used: the proportional mode and the incremental mode. The proportional actuation consists to control the deformation of a smart material such as piezoelectric material, shape memory alloys (SMA) and active polymers. This deformation allows generating a continuous displacement of the microrobot [1], [2]. The use of smart materials to actuate microrobots provides better performances than the use of conventional actuators because they are more suitable and compatible for miniaturization. However, despite their intrinsic high resolution, these materials have some drawbacks, making the design of efficient microrobots a hard task. Their behavior is often nonlinear and usually non-stationary (environmental conditions as temperature and humidity have a great influence on their static and dynamic

\footnotetext{
1 are with Université Bourgogne Franche-Comté, FEMTO-ST Institute/CNRS, Besançon, France.

2 are with Université de Montpellier, LIRMM/CNRS, Montpellier, France.
}

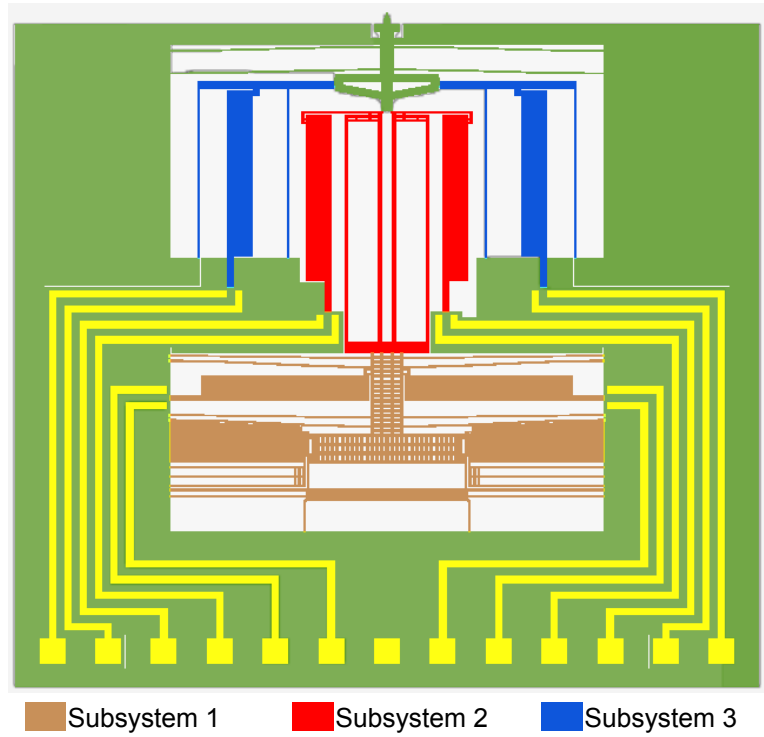

Fig. 1. Multistable module architecture including three subsystems, support and conductive lines. The module has a width of $12 \mathrm{~mm}$ and a high of $11 \mathrm{~mm}$.

behaviors) [3]. Under these conditions, their control becomes complex and requires the integration of very small sensors and the use of bulky and expensive instruments for signal processing and real-time operating. In contrast, the incremental actuation mode allows a discrete linear displacement of the microrobot without closed loop control. As described in the literature, most of these actuators are based on a bistable mechanism [4]-[7]. Switching between two states, such mechanism guarantees a mechanical stability of the states in open loop without energy consumption unlike stickslip [8], inertial [9] or inchworms actuators [10], [12]. Other discrete actuators were presented in the literature such as tristable [11] and quadristable [13] actuators, but their displacement range is limited since it depends directly on the stable positions. To reach more stable positions, it is possible to connect several bistable modules in serial [14] or parallel distribution [15]. Nevertheless, this option increases the size of the actuator and its microfabrication becomes ever more complex and non-intuitive. To tackle this limitation, a new multistable module has been developed to reach several stable positions within a miniaturized structure [16]. This new architecture allows switching linearly a moving part between several stable positions in one dimensional direction. It presents many advantages such as: Monolithic compact structure with more discrete position, low cost, low 


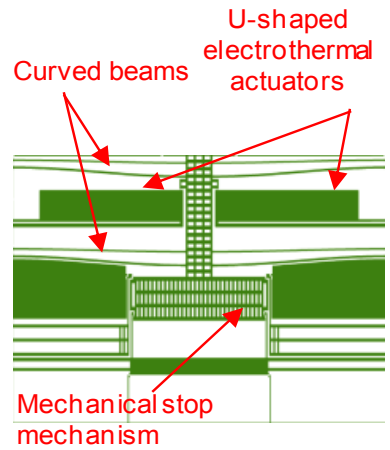

(a)

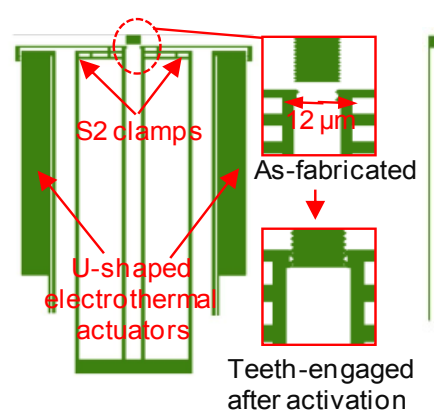

(b)

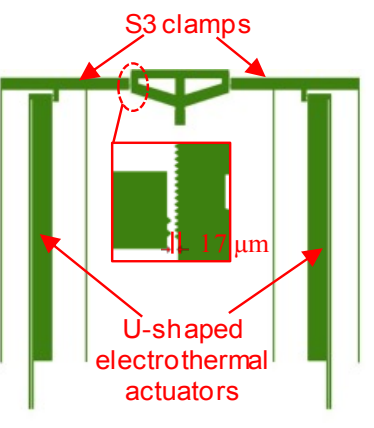

(c)

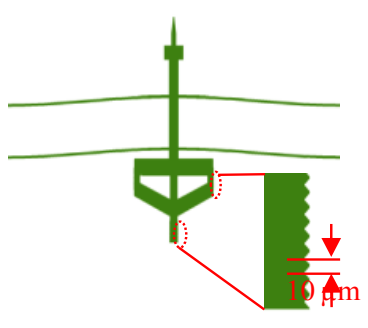

(d)

Fig. 2. Subsystems and moving part illustration. (a) Subsystem S1, (b) Subsystem S2, (c) Subsystem S3 and (d) Moving part.

energy consumption (no energy is needed to hold the moving part at rest) and open loop control (no sensors are needed) with low sensitivity to noise. Following the experimental validation reported in [16], we propose here to validate the performances of the fabricated multistable modules in terms of repeatability and reproducibility. This validation is mandatory to demonstrate the effectiveness of the module as it is expected to be an elementary component of the next generation of digital microrobots. To this end, a series of experimental measurements are conducted on individual and multiple modules. Each series consists on a control sequence generating 12 upward steps followed by 12 downward steps. All the generated displacements are compared with the theoretical displacements and a statistical analysis is carried out to assess the repeatability and reproducibility. The results analysis shows a good agreement between the theoretical and the experimental displacements. In other words, the multistable prototype is able to reach 13 (from position 0 to position 12) stable positions linearly in one dimensional direction with a step of about $10 \mu \mathrm{m}$. These capabilities open a promising perspectives and applications of this module to achieve microrobotics tasks.

The paper is organized as follows. In section II, a brief reminder about the multistable design architecture and its functioning principle are reported. Section III, describes the fabrication process of the multistable module. A series of experimental measurements and their analysis in terms of repeatability and reproducibility are presented in section IV. The results are discussed in section V. Finally, section VI summarizes the work and gives some promising perspectives.

\section{Multistable module}

This section reminds briefly the architecture of the proposed multistable and its principle of functioning. Instead of using multiple bistables, this compact structure is based on three subsystems allowing to generate several stable positions.

\section{A. Mechanical design}

Figure 1 shows the architecture of the multistable module described in our previous work [16], [17]. It consists of three subsystems acting on a moving part. Each subsystem is composed of two U-shaped electrothermal actuators and flexible structures. These subsystems ensure the switching, holding guiding of the moving part at stable positions. For brevity, no distinction is made between flexible structures of subsystems and clamps.

The subsystem 1, illustrated in Fig 2.a, consists of a shuttle connected to S2 clamp. Actuated by two U-shaped electrothermal actuators, its movement is guided by curved beams and a mechanical stop. Its main function is to move vertically the $\mathrm{S} 2$ clamps with a defined and repeatable displacement. The second subsystem consists of two clamps actuated by two U-shaped electrothermal actuators. It allows opening the S2 clamp. These clamps are designed to be normally closed after the manual activation phase (post fabrication activation). Designed as a gantry, this subsystem ensures the horizontal entry and exit of the teeth as illustrated in Fig. 2.b. Initially, the S2 clamp is opened and then moved vertically to the lower stop position. This vertical displacement engages the couple of teeth on the head of each side of the clamp with the first couple of teeth on the moving part. Likewise, the subsystem S3 uses two U-shaped electrothermal actuators. They act on the S3 clamp based on a set of two gantries as shows in Fig 2.c. Its function consists to close the $\mathrm{S} 3$ clamp in order to hold the moving part during stepping of the multistable actuator. Unlike, the previous subsystems, it does not require any initialization since it is normally open. The three subsystems act in the final step on a moving part which is based on a butterfly shape (see Fig. 2.d). This part reduces the horizontal extension of the gantries of clamp S3. To maintain this part and to guide its vertical displacement, a couple of curved beams are used as shown in Fig. 2.d.

\section{B. Principle of functioning}

The functioning principle of the multistable module revolves around a specific sequence of several simple steps in order to perform a displacement such as moving, opening and/or closing the clamps with and without the moving part. This sequence allows switching the moving part between several stable positions linearly in one dimensional direction. 


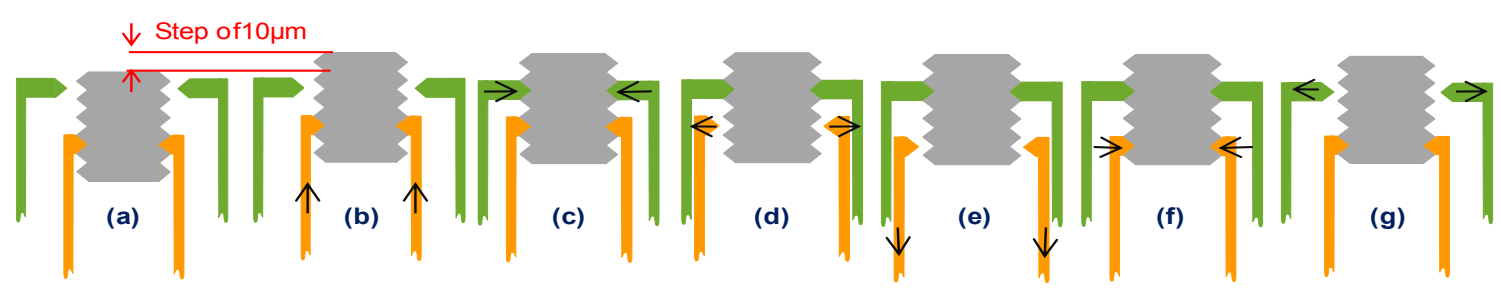

Fig. 3. Sequence order to make an upward step. (a) Rest configuration, (b) Firstly, S2 clamp moves upward with holding the moving part, (c) S3 clamp holds the moving part, (d) S2 clamp releases the moving part, (e) S2 clamp moves downward and (f) S2 clamp holds the moving part at bottom position of S1, (g) Finally, S3 clamp releases the moving part.

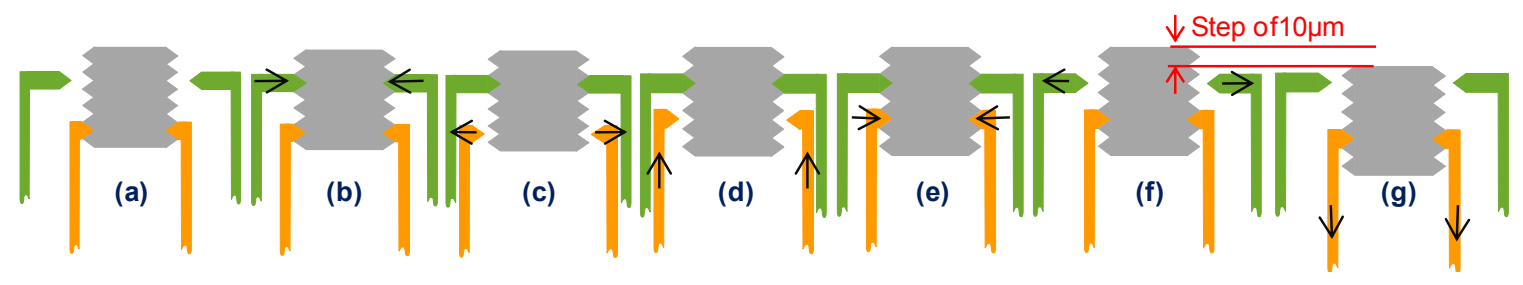

Fig. 4. Sequence order to make a downward step. (a) Rest configuration (b) Firstly, S3 clamp holds the moving part, (c) S2 clamp releases the moving part, (d) S2 clamp moves upward and (e) S2 clamp closes in the upper position, (f) then S3 clamp releases the moving part, (g) finally, S2 clamp drives down the moving part.

The sequence order to make upward and downward steps of the moving part are given in Fig. 3 and Fig. 4 respectively.

All the module actuator's are involved in these sequences. They generate the displacements and forces that are required to move, open or close the clamps. For each step, a specific control sequence must be applied. Figure 5 illustrates the control sequences that give rise to upward and downward steps respectively. We will see further that these figures correspond to the experimental optimized control sequences.

\section{MICROFABRICATION}

As described in [16], the multistable module was fabricated using a single-crystalline silicon-on-insulator (SOI) wafer. As shown in Fig. 6a, this wafer is composed of four layers: (i) a $100 \mu \mathrm{m}$ thick silicon layer dedicated to the active area, (ii) a $2 \mu \mathrm{m}$ thick silicon dioxide insulation layer, (iii) a $380 \mu \mathrm{m}$ thick handle silicon layer and (iv) a $2 \mu \mathrm{m}$ thick silicon dioxide layer serves as a hard mask. To perform the module fabrication several operations were performed based on clean room facilities. The process began by a dry etching of the wafer backside (see Fig. 6b). Then a dry etching was performed on the silicon layer (wafer front side) as illustrated in Fig. 6c. Following, the silicon backside layer (handle layer) was etched (see Fig. 6d). After that, the wafer was dived into a Hydrofluoric acid (HF 49\%) solution in order to release the useless parts from the wafer (see Fig. 6e). The HF solution etches transversely the buried silicon dioxide located between the device and handle openings. The final operation consists to deposit a gold layer of $200 \mathrm{~nm}$ thick through a shadow mask (see Fig. 6f). The resulted prototype is shown in Fig. 7. After manual activation, the prototype was glued on a PCB and an electrical connections between the module and the PCB pads were made trough a wire bonding.
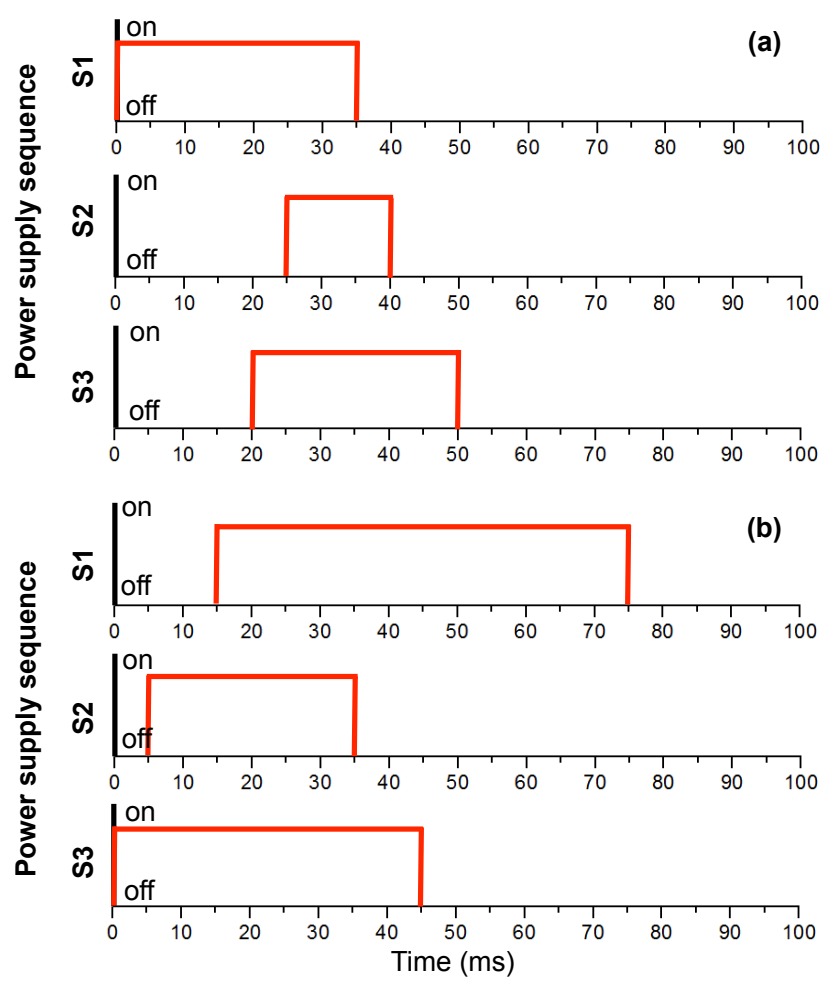

Fig. 5. Control sequence of the U-shaped actuators. (a) Upward step control sequence, (b) Downward step control sequence.

\section{EXPERIMENTAL CHARACTERIZATION}

This section deals with the validation of the performances of the multistable prototype in terms of repeatability and reproducibility. To this purpose, a series of experimental measurements are conducted on individual and multiple modules. Then, a statistical analysis based on ANOVA 
(a)

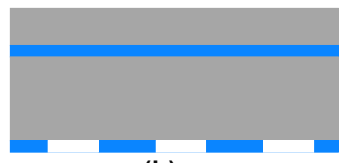

(b)

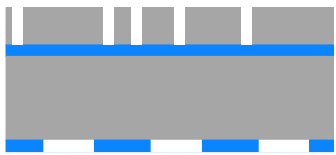

(c)

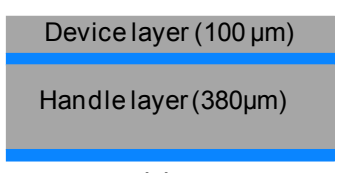

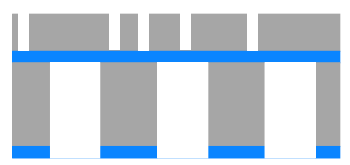

(d)

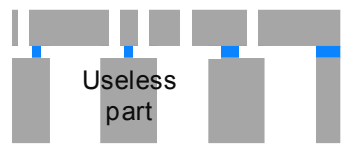

(e)
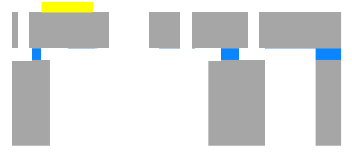

(f)
Silicon Thermal SiO2 $(2 \mu \mathrm{m})$

Fig. 6. Fabrication process of the multistable module on SOI wafers.

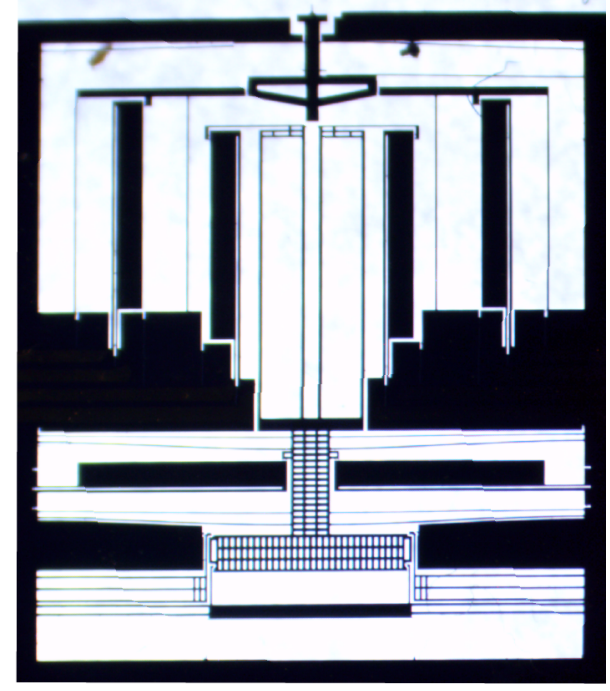

Fig. 7. Micro-fabricated multistable module.

(analysis of variance) is performed.

\section{A. Experimental Bench}

The experimental bench employed in this study is depicted in Fig. 8. It is composed of a PCB carrying the prototype, an electronic circuit and a MEMS analyzer (MAS-500 from Polytec company). To ensure the most favorable conditions, the PCB is placed on an anti-vibration table. It is also dissociated from the electronic circuit to avoid any vibrations that may come from the relays switching movements. To supply the different actuators, the PCB is connected to the electronic circuit. Based on a microcontroller, this circuit controls the supply time of the control sequences of the U-shaped actuators given in Fig. 5. The circuit is also equipped with connectors and relays. Each relay allows switching on/off the current of an electrothermal actuator stage. Otherwise, buttons are added to switch control between upward and downward steps. The prototype displacements are captured via the MEMS analyzer (MSA-500) which is able the mea-

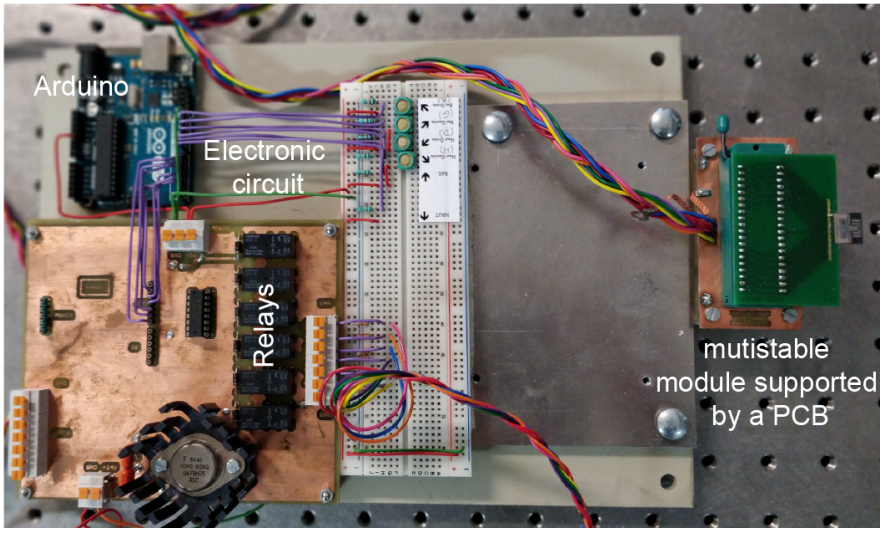

Fig. 8. Experimental Bench.

sure nanometric displacements in an ambient environment using the planar motion analysis software. Before starting the experiments, the supply times of each electrothermal actuator are optimized experimentally. Indeed, the mechanical displacements, of each subsystem, are characterized under different frequencies of square voltage signals of 20 volts, during heating (power on) and cooling cycles (power off). These displacements are recorded thanks to the use of a highspeed camera with a high rate frame record. Then a treatment under pixel tracking software is achieved. The chronograms of U-shaped electrothermal actuators given in Fig. 5 are obtained.

\section{B. Repeatability analysis}

As reported in the introduction, the proposed module is expected to generate a regular discrete displacement with a step of $10 \mu \mathrm{m}$. The repeatability analysis aims to asses experimentally this property of the module. To guarantee the most favorable conditions of the analysis, the set of conditions reported in [18] are respected: same measurement procedure, same operator, same measurement system (MSA500), same operating condition, same location and replicate measurements on the same device. Following this procedure, we performed a 10 series of measurements on an individual module. Each series consists of a control sequence generating 12 upward steps and 12 downward steps. For practical reasons, the delay between each sequence is set at $200 \mathrm{~ms}$. All the measure are captured by a MEMS analyzer (MSA500) able to measure nanometric displacements. For brevity, only the measurements during the upward displacements are reported in Tab. I. It is worth to note that these measurements are considered as independent observations. To highlight the step displacements of the module, a subtraction is made between each successive positions. Then a one way ANOVA analysis is performed under Matlab software. It allows to asses the differences among individual series of measurements and the difference between means of the 10 series. The obtained result reveals a p-value of 0.9994 and a mean square (ms) of the error of 0.01429 . The first value, very close to 1 , indicates that there is no significant difference between the means of each series. Whilst, the $\sqrt{\text { mean square error }}$ 
TABLE I

EXPERIMENTAL MEASUREMENTS REPRESENTING 10 SERIES OF 12 UPWARD STEPS PERFORMED ON AN INDIVIDUAL MODULE. ALL THE DISPLACEMENTS ARE IN $\mu \mathrm{M}$ UNIT.

\begin{tabular}{|c|c|c|c|c|c|c|c|c|c|c|}
\hline $\begin{array}{ll} & \text { series Nbr. } \\
\text { Position Nbr. } & \end{array}$ & 1 & 2 & 3 & 4 & 5 & 6 & 7 & 8 & 9 & 10 \\
\hline 1 & 0 & 0 & 0 & 0 & 0 & 0 & 0 & 0 & 0 & 0 \\
\hline 2 & 10.19 & 10.2 & 10.17 & 10.06 & 10.18 & 10.08 & 10.01 & 10.04 & 10.06 & 10.17 \\
\hline 3 & 20.3 & 19.99 & 20.17 & 20.17 & 19.93 & 20.02 & 20.17 & 19.92 & 20.01 & 20.15 \\
\hline 4 & 30.15 & 30.19 & 29.98 & 30.38 & 29.99 & 30.05 & 30.35 & 30.01 & 30 & 30.31 \\
\hline 5 & 40.32 & 40.27 & 40.13 & 40.39 & 40.13 & 40.18 & 40.35 & 40.15 & 40.19 & 40.25 \\
\hline 6 & 50.44 & 50.34 & 50.33 & 50.31 & 50.32 & 50.34 & 50.3 & 50.2 & 50.33 & 50.27 \\
\hline 7 & 60.3 & 60.34 & 60.33 & 60.33 & 60.31 & 60.31 & 60.35 & 60.1 & 60.29 & 60.31 \\
\hline 8 & 70.57 & 70.39 & 70.47 & 70.6 & 70.49 & 70.47 & 70.54 & 70.43 & 70.41 & 70.53 \\
\hline 9 & 80.56 & 80.46 & 80.47 & 80.7 & 80.53 & 80.4 & 80.49 & 80.44 & 80.36 & 80.56 \\
\hline 10 & 90.6 & 90.51 & 90.54 & 90.56 & 90.46 & 90.53 & 90.69 & 90.47 & 90.49 & 90.54 \\
\hline 11 & 100.8 & 100.7 & 100.46 & 100.85 & 100.52 & 100.67 & 100.73 & 100.48 & 100.57 & 100.8 \\
\hline 12 & 110.81 & 110.85 & 110.71 & 110.83 & 110.56 & 110.65 & 110.91 & 110.62 & 110.52 & 110.68 \\
\hline 13 & 120.75 & 120.76 & 120.72 & 120.69 & 120.63 & 120.68 & 120.82 & 120.59 & 120.53 & 120.69 \\
\hline
\end{tabular}

indicates a repeatability of $0.1195 \mu \mathrm{m}$. This means that the majority of the achieved steps are expected to be within $0.1195 \mu \mathrm{m}$ of the theoretical step of $10 \mu \mathrm{m}$. This result demonstrates the capacity of the module to generate regular discrete steps of $10 \mu \mathrm{m}$.

\section{Reproducibility analysis}

In this study, reproducibility refers to the degree of agreement between the experimental measurements carried out by one operator on different multistable modules using the same measurement system. In other words, it represents the ability of a module to replicates the displacements of another module (modules could come from the same wafer/run process or from different wafers). To assess this property, we used four modules coming from the same wafer. Respecting the above-mentioned conditions, a four series of 12 upward and downward steps are conducted on each module. Likewise the previous experience, the delay between each sequence is set at $200 \mathrm{~ms}$. All the measurements are recorded by a MSA-500 MEMS analyzer able to capture nanometric displacements. For brevity, only the measurements during the upward displacements are reported in Tab. II. To extract the generated step displacements of each module, a subtraction is made between each successive positions for each series. Then a one way ANOVA analysis is performed under Matlab software in order to analysis the differences between the module's steps. The obtained result reveals a $\mathrm{p}$-value of 1 which means that there is no significant differences between measurements. It reveals also a mean square error of 0.033 . This means that the majority of the achieved steps by the four modules are expected to be within $0.1817 \mu \mathrm{m}$ (square root of the (ms) value) of the theoretical step of $10 \mu \mathrm{m}$. This result demonstrates the ability of any module to replicates the displacement of others modules.

\section{DISCUSSION}

The performed analysis in this work indicates that the proposed modules can generate regular discrete steps of about $10 \mu \mathrm{m}$. The repeatability analysis of one module leads to a value of $0.1369 \mu \mathrm{m}$. Meanwhile, the reproducibility analysis leads to a value of $0.1817 \mu \mathrm{m}$ which is slightly larger than the previous value. These values give the interval variation of the generated steps for one and four modules respectively. These values are largely acceptable but room is left for improvement especially in regards to the fabrication process. Indeed, the small variations that can be observed between the experimental and theoretical displacements may come from the tolerances resulting from the fabrication process. It is worth to notice that the distance between teeth on the moving part and the latches (top to top or bottom to bottom) is independent to some level from the fabrication tolerances. Considering the same fabrication conditions on the local area of the teeth on the wafer, the excess or insufficient etching of the teeth patterns is the same for the different teeth. In result, the teeth faces are more or less etched, however the distance between them remains the same. Thereby, one reason of using the teeth in the multistable module was to increase the accuracy of the steps. This hypothesis was validated on the fabricated prototypes where the distance between two successive teeth in the moving part is measured at $10 \mu \mathrm{m}$. Besides, the mean of the shuttle displacement in subsystem $\mathrm{S} 1$ is about $10.3 \mu \mathrm{m}$. The difference between the displacement of the shuttle in subsystem S1 and the distance between two successive teeth could affect the repeatability of the multistable module and may cause a backlash between the teeth after an up/downward displacement. The resulted backlash may generate or not a random slipping of the clamps teeth on the moving part teeth. The appearance of this random slipping may explain the values obtained during the repeatability and reproducibility analysis.

\section{CONCLUSION}

In this paper, an experimental characterization of a new multistable module is addressed. Devoted to MEMS actuation, in particular digital microrobotics, this module is based on an original architecture that combines three subsystems. This structure presents several advantages comparing to bistable modules: monolithic compact structure with several discrete position, low cost, low energy consumption and open loops control (no sensors are needed). It is worth to notice 
TABLE II

EXPERIMENTAL MEASUREMENTS REPRESENTING 16 SERIES OF 12 UPWARD STEPS PERFORMED ON FOUR MODULES, FOUR SERIES FOR EACH MODULE. ALL THE DISPLACEMENTS ARE IN $\mu$ M UNIT.

Position Nbr.

\begin{tabular}{|c|l|c|c|c|c|c|c|c|c|c|c|c|c|c|}
\multicolumn{2}{c|}{ Module } & 1 & 2 & 3 & 4 & 5 & 6 & 7 & 8 & 9 & 10 & 11 & 12 & 13 \\
\hline module 1 & series 1 & 0 & 10.06 & 20.01 & 30 & 40.19 & 50.33 & 60.29 & 70.41 & 80.36 & 90.49 & 100.57 & 110.52 & 120.53 \\
& series 2 & 0 & 10.17 & 20.15 & 30.31 & 40.25 & 50.27 & 60.31 & 70.53 & 80.56 & 90.54 & 100.8 & 110.68 & 120.69 \\
& series 3 & 0 & 9.98 & 19.86 & 30.1 & 40.21 & 50.21 & 60.31 & 70.58 & 80.3 & 90.67 & 100.65 & 110.57 & 120.58 \\
& series 4 & 0 & 10.11 & 20.12 & 30.33 & 40.4 & 50.56 & 60.38 & 70.64 & 80.48 & 90.4 & 100.65 & 110.6 & 120.66 \\
\hline module 2 & series 1 & 0 & 10.23 & 20.28 & 30.3 & 40.46 & 50.58 & 60.7 & 70.65 & 80.8 & 90.87 & 101.06 & 110.95 & 120.98 \\
& series 2 & 0 & 10.19 & 20.32 & 30.32 & 40.44 & 50.61 & 60.78 & 70.72 & 80.87 & 90.82 & 101.06 & 110.98 & 121.1 \\
& series 3 & 0 & 10.26 & 20.27 & 30.23 & 40.47 & 50.61 & 60.76 & 70.66 & 80.82 & 90.8 & 101.11 & 110.99 & 120.98 \\
& series 4 & 0 & 10.22 & 20.34 & 30.24 & 40.48 & 50.65 & 60.76 & 70.7 & 80.86 & 90.85 & 101.08 & 110.96 & 121.04 \\
\hline module 3 3 & series 1 & 0 & 10 & 19.97 & 30.41 & 40.09 & 50.1 & 60.63 & 70.87 & 80.78 & 90.87 & 101.08 & 110.85 & 120.94 \\
& series 2 & 0 & 10.14 & 20.07 & 30.54 & 40.31 & 50.27 & 60.58 & 70.67 & 80.63 & 91.01 & 101.15 & 110.79 & 120.69 \\
& series 3 & 0 & 10.21 & 20.07 & 30.57 & 40.35 & 50.31 & 60.59 & 70.67 & 80.72 & 91.05 & 101.15 & 110.89 & 120.75 \\
& series 4 & 0 & 10.14 & 20.15 & 30.57 & 40.33 & 50.31 & 60.67 & 70.74 & 80.72 & 91.03 & 101.13 & 110.82 & 120.74 \\
\hline module 4 4 & series 1 & 0 & 10.16 & 20.27 & 30.44 & 40.61 & 50.89 & 60.8 & 71 & 80.76 & 91.16 & 100.91 & 111 & 120.5 \\
& series 2 & 0 & 10.19 & 20.3 & 30.48 & 40.65 & 50.87 & 60.81 & 71 & 80.77 & 90.95 & 100.82 & 110.93 & 120.72 \\
& series 3 & 0 & 10.22 & 20.15 & 30.55 & 40.62 & 50.75 & 61 & 70.8 & 80.83 & 91.01 & 100.92 & 110.95 & 120.78 \\
& series 4 & 0 & 10.2 & 20.32 & 30.44 & 40.79 & 50.73 & 60.8 & 70.7 & 80.78 & 91.14 & 100.99 & 110.8 & 120.67 \\
\hline
\end{tabular}

that this design is made also to achieve a theoretical discrete displacement step of $10 \mu \mathrm{m}$. To assess this property from experimental point of view, repeatability and reproducibility analysis of the proposed multistable is carried out. Basically, a series of experimental measurements are conducted on individual and multiple modules. Each series consists on a control sequence generating 12 upward steps followed by 12 downward steps. Then, a statistical analysis based on one way ANOVA is performed. The results coming from this analysis indicate a good agreement between the experimental and theoretical steps. As a result, the multistable prototype is able to reach 13 stable positions linearly in one dimensional direction with a step of about $10 \mu \mathrm{m}$. This capacity of generating regular displacements shows a great promise and could benefit to several applications related to MEMS devices (micro-actuators, micro-positioners, microrobots, etc).

Ongoing work includes the process fabrication improvement. Indeed, significant decrease of fabrication tolerances will further increase the module's ability to generate regular displacement steps. Future work is also concerned with the combination of several multistable modules to design new and innovative microrobot architectures.

\section{ACKNOWLEDGMENT}

This work has been supported by the Labex ACTION project (con-tract ANR-11-LABX-01-01), the French RENATECH network and its FEMTO-ST technological facilities.

\section{REFERENCES}

[1] Régnier S, and Chaillet N, "Microrobotics for Micromanipulation", Wiley-ISTE, publisher, 2010.

[2] Rakotondrabe M, Haddab Y, Lutz P, "Quadrilateral modeling and robust control of a nonlinear piezoelectric cantilever", IEEE Trans Control Syst Technol 17 (T-CST) 17(3):528-539, 2009.

[3] M. Rakotondrabe, C. Clevy and P. Lutz, "Hysteresis and vibration compensation in a nonlinear unimorph piezocantilever", in IEEE/RSJIROS (International Conference on Intelligent Robots and Systems), Nice, France, pp. 558-563, 2008.
[4] Y. Wu, G. Ding, C. Zhang, J. Wang, S. Mao, and H. Wang, ”Design and implementation of a bistable microcantilever actuator for magnetostatic latching relay," Microelectron. J., vol. 41, no. 6, pp. 325-330, Jun. 2010.

[5] Q. Chen, Y. Haddab, and P. Lutz, "Microfabricated bistable module for digital microrobotics," J. Micro-Nano Mechatron., vol. 6, no. 12, pp. 1-12, Feb. 2011.

[6] Q. Chen, Y. Haddab and P. Lutz "Characterization and control of a monolithically fabricated bistable module for microrobotic applications", in IEEE/RSJ- IROS (International Conference on Intelligent Robots and Systems), pp. 5756-5761, 2010

[7] H. Hussein, V. Chalvet, P. LeMoal, G. Bourbon, Y. Haddab and P. Lutz, "Design optimization of bistable modules electrothermally actuated for digital microrobotics", IEEE/ASME International Conference on In Advanced Intelligent Mechatronics (AIM), pp. 1273-1278, 2014.

[8] Rakotondrabe M, Haddab Y, Lutz P, "Voltage/frequency proportional control of stick-slip microsystems". IEEE Trans Control Syst Technol (T-CST) 16(6):1316-1322, 2008.

[9] R. Bchi, W. Zesch, A. Codourey et R. Siegwart, "Inertial drives for micro- and nanorobots: Analytical study", SPIE's, Intelligent Systems and Advanced Manufacturing, Philadelphia, USA, 23-26 octobre 1995.

[10] Chen IM, Gao SH "Locomotive gait generation for Inchworm like robots using finite state approach", Robotica 19(5):535-542, 2001.

[11] G. Chen, Q. T. Aten, S. Zirbel, B. D. Jensen, and L. L. Howell, "A Tristable Mechanism Configuration Employing Orthogonal Compliant Mechanisms", J. Mech. Robot., vol. 2, no. 1, pp. 014501-014501-6, Nov. 2009.

[12] D. S. Contreras and K. S. J. Pister, "Dynamics of electrostatic inchworm motors for silicon microrobots", 2017 International Conference on Manipulation, Automation and Robotics at Small Scales (MARSS), Montreal, QC, 2017, pp. 1-6.

[13] J. S. Han, C. Mller, U. Wallrabe, and J. G. Korvink, ”Design, Simulation, and Fabrication of a Quadstable Monolithic Mechanism With X- and Y-Directional Bistable Curved Beams", J. Mech. Des., vol. 129, no. 11, pp. 1198-1203, Nov. 2006.

[14] Q. Chen, Y. Haddab and P. Lutz "Digital Microrobotics Based onBistable Modules : Design of Compliant Bistable Structures", in IEEE/ASME International Conference on Mechatronics and Embedded Systems and Applications, Beijing, ZH, 2008, pp. 36-41

[15] V. Chalvet, Y. Haddab, and P. Lutz, "A Microfabricated Planar Digital Microrobot for Precise Positioning Based on Bistable Modules", IEEE Trans. Robot., vol. 29, no. 3, pp. 641-649, Jun. 2013.

[16] H. Hussein, I. Bouhadda, A. Mohand-Ousaid, G. Bourbon, P. L. Moal, Y. Haddab, and P. Lutz "Design and fabrication of novel discrete actuators for microrobotic tasks", Sensors and Actuators A: Physical, Volume 271, Pages 373-382, 2018.

[17] I. Bouhadda, A. Mohand-Ousaid, P. Le Moal, G. Bourbon and P. Lutz, "Dynamic characterization of an electrothermal actuator devoted to discrete MEMS positioning", 19th Symposium on Design, Test, Integration and Packaging of MEMS/MOEMS (DTIP), pp.1-4, 2017. 
[18] JCGM guidance document, "International vocabulary of metrology Basic and general concepts and associated terms (VIM)", 3rd edition, JCGM 2012 